\title{
Interactions Between Root and Shoot Competition and Plant Traits
}

\author{
James F. Cahill and Eric G. Lamb \\ Department of Biological Sciences, University of Alberta, Edmonton, Alberta, Canada T6G 2E9
}

\begin{abstract}
A plant's performance depends on its ability to deal with numerous, simultaneous ecological challenges. In both natural and production systems, dominant challenges include competition for soil resources and light, herbivory, and general abiotic stress. A central goal of research is to understand how these processes interact with each other and with plant phenotype (above- and belowground) to influence overall plant performance. Complicating these efforts is the reality that plants are phenotypically plastic with the phenotypic response to one challenge potentially altering the impact of a different challenge. Furthermore, factors external to the plant (e.g., the genotypic and phenotypic composition of the surrounding plants) can also influence the consequence of various ecological pressures. We have been using Arabidopsis thaliana as a model organism to help disentangle this complicated web of ecological interactions. Competitive ability can be influenced by small genotypic changes. A plant's ability to suppress competitors is driven mainly by size-related traits and soil fertility and a plant's ability to withstand harm coming from numerous sources. The relative importance of competition is contingent not only on the match between genotype and environment, but also on the diversity of genotypes within a given population. There is a need to consider alternative effects of plant traits along with the cascading consequences of plant responses to biotic and abiotic challenges.
\end{abstract}

The central problem in plant community ecology is that the processes that govern community structure and function are extremely complicated, particularly in comparison with the relatively "simple" factors that influence the performance of a single species. The main theme of our research is to try to understand how species traits and ecological processes interact to influence the diversity and abundance of species. To do this involves studies of physiology, evolutionary ecology, herbivory, competition, and multitrophic interactions. This is further complicated by the recognition that these factors are themselves dependent on variable climatic conditions and nutrient inputs. In this article, we summarize some recent advances addressing questions including the traits associated with competitive ability in plants, the costs and benefits of plasticity in those traits, and how plants respond to multiple challenges such as competition and herbivory. These questions are at the foundation of important agricultural issues such as genetically engineered crops, productivity, and weed suppression.

A wide range of factors, including herbivory, mycorrhizal associations, competition, and pathogens, can simultaneously influence the growth of an individual plant (Grime, 2001; Keddy, 2001). Ultimately, a plant's performance will be determined by how it responds to all of these factors in addition to microenvironmental conditions and general resource supplies (Fig. 1). Individual plants are the focus of natural selection, and thus the phenotype of a plant should reflect adaptations to these ecological challenges. These adaptations will be balanced by

This work was supported by funding from a NSERC Discovery grant awarded to JFC

We thank Jim Syvertsen and ASHS for the opportunity to present this talk at the 2006 annual meeting in New Orleans, LA. some inherent constraints, or tradeoffs, governed by the reality that if a plant invests a proportion of a finite energy supply to one function, there is less to allocate to other functions (Grime, 2001; Tilman, 1990). For example, a plant needs to be taller to compete for light, but to be taller, the roots first have to take up sufficient nutrients to build the required stems and leaves. Being taller also has costs such as vulnerability to water stress or lodging. Allocating energy and adjusting phenotype toward one function has costs that can make a plant less able to deal with other ecological factors. In this article, we focus on competition and show how a wide range of complex interactions influence what was once thought to be a very straightforward process.

The classic view of competition is of a direct interaction or fight, but in plants, competition is generally indirect with one plant hurting another by taking up limited resources (resource competition) that another plant could otherwise access (Keddy, 2001). Direct competition only occurs through relatively rare mechanisms such as allelopathy (Williamson, 1990). Even resource competition is complex because in natural systems, multiple resources are often simultaneously limiting. For example, root and shoot competition can both greatly reduce plant growth. Root and shoot competition are not independent processes because root competition for limited water and nutrients can reduce a plant's ability to compete for light by stunting initial shoot growth (Cahill, 1999, 2002). In addition, there are two general strategies of competition in plants (Goldberg, 1990). An effect competitor is a plant that is good at denying neighbors access to limiting resources, whereas a response competitor is one that avoids harm from its neighbors.

At the broadest scale, we can ask a very simple question straight from "The Origin of Species" (Darwin, 1859). Darwin said that more closely related species should compete more strongly than distantly related species because more closely related species are more similar in their traits and requirements. To test this proposition (Cahill et al., unpublished data), we used a meta-analysis of competition experiments in which the competitive effect of one species on another, or the degree of growth reduction compared with a control plant grown alone, had been measured. We developed a phylogeny and measured the phylogenetic distance (millions of years since evolutionary divergence) between each species pair. We found a significant relationship between competition intensity and phylogenetic distance, supporting Darwin's prediction that closely related species did compete more intensely than distantly related ones. This relationship, however, explained very little of the variation in our data. Looking further, we found that the intensity of competition when monocots only competed against monocots and dicots only competed against dicots was very similar. However, when dicots competed against monocots and monocots competed against dicots, the monocot was always the poorer competitor. This suggested that dicots have some trait(s) that give them a significant competitive advantage over monocots.

What are the traits that can confer competitive ability? A number of comparative studies have shown that plant size is the main indicator of competitive ability (Gaudet and Keddy, 1988, 1995; Rösch et al., 1997). It is clear that big plants can win over little plants, but these studies were done under fertile conditions in which the plants competed primarily for light. It remains unclear whether large size confers enhanced competitive ability in systems where plants must compete belowground for soil-limited resources. For example, in native rough fescue grasslands in Alberta, more than $80 \%$ of the biomass is belowground and there is intense competition for soil resources but almost none for light (Lamb et al., 2007). In this system, tiny 


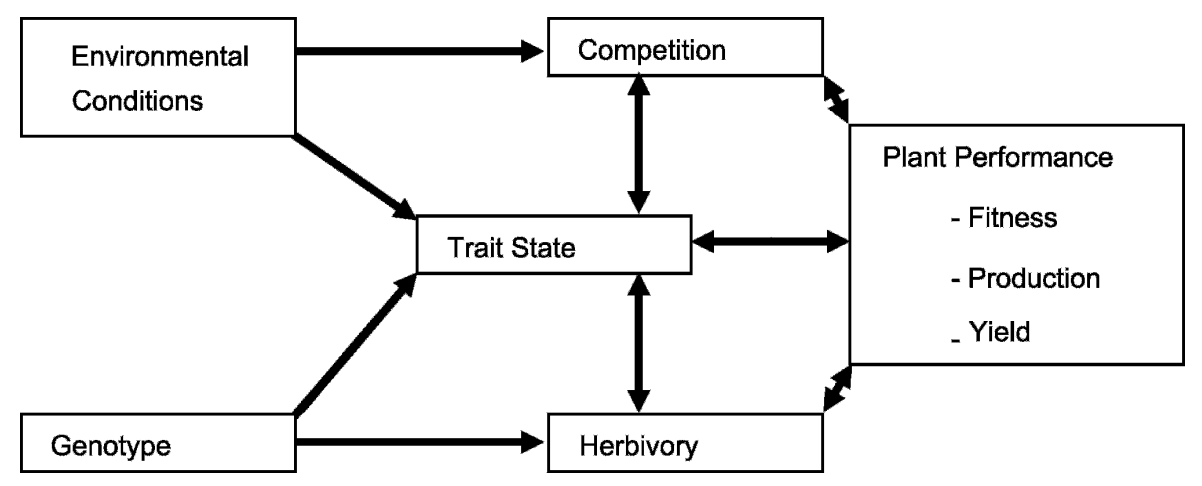

Fig. 1. Plant performance (fitness, productivity, or yield) is affected by numerous interacting factors, including plant genotype and general environmental conditions, competition, and herbivory. In addition to direct effects, ecological factors such as competition and herbivory can cause plants to modify their trait states through phenotypic plasticity.

seedlings had competitive ability equivalent to mature plants many times their size (Lamb and Cahill, 2006). Similarly, belowground competitive ability did not appear to be linked to size, because the intensity of root competition experienced by the seedlings of 10 species was unrelated to the mass of the plant's root system (Cahill, 2003).

What traits confer belowground competitive ability? Our laboratory has recently focused efforts on bringing the extensive work by plant geneticists and developmental biologists into an ecological context. The approach is simple; we compared mutants of Arabidopsis thaliana with abnormal leaf or root morphology and physiology that differed in a single gene from wild-type Columbia (Cahill et al., 2005; Kembel, 2006). Mutants were grown in competition against Columbia with high or low nutrient conditions. Competition was exclusively belowground in the low nutrient treatments and there was a combination of above- and belowground competition in the high nutrient treatments. Competitive effect, or the ability for one plant to harm another, was largely based on size; however, competitive response, or the ability to avoid harm, could not be explained by plant size. Instead, we found that competitive response was a function of the specific phenotypes involved. We do not know why some phenotypes did better than others, although we suspect that ethylene is involved for a variety of reasons. More specifically, the Columbia genotype experienced no measurable competition only against the ethylene-overproducing genotype. One possible explanation could be that ethylene serves as a signal for competition, although substantially more work will need to be done to test this hypothesis. Ongoing work is examining the relationships between these phenotypes, competition, and plant fitness (Kembel, 2006). The most important finding thus far is that there was no correlation between competitive effect and competitive response indicating that at a genetic level, they are fundamentally different traits. This separation has important implications for the evolution of competitive ability, particularly in managed systems in which, challenge for ecologists because changing one piece can have unexpected consequences for the whole system. Some ecologists have begun to address this complexity directly through statistical methods that allow all of the processes in a system to be simultaneously described (Grace, 2006; Shipley, 2000). In our laboratory, we have taken an experimental approach using the Arabidopsis system to examine whether there are genotypes adapted to withstand multiple ecological stressors (Cahill and Keddie, unpublished data). We measured competitive response, the ability to withstand nitrogen stress, and the tolerance of herbivory by Trichoplusia ni in 47 lines of Arabidopsis, including both mutants and ecotypes. There were three major groups among the 47 lines: strong effect competitors, plants very resistant to herbivory, and strong response competitors that were also very stress-tolerant. This suggests that there have been selective pressures on these plants in at least three directions. In a second large experiment, we tested whether there was genetic specialization to suites of ecological factors by manipulating density (competition), the genetic diversity of competitors, herbivory, and fertilization. The results were complex, but as a whole appear to indicate that plants do specialize on suites of ecological conditions. For example, some genotypes specialized on resisting herbivory, and those genotypes are most advantageous when neighboring plants are also of that genotype.

A central goal of community ecology is to understand how ecological processes structure communities. Similarly, in an applied setting, the goal is to understand how to maximize yield or production. The goals may be different but the underlying processes are the same. Unfortunately, even relatively simple processes like plant competition have been studied for hundreds of years with surprisingly little progress (Keddy, 2001). To move forward, we need to move past simple experiments in which we vary the degree of competition and find that competition suppresses plants. Clearly, individual plant traits and phenotype are important, but there is a great deal to learn, particularly about root competition. These traits have a genetic basis and understanding the link between genotype and competitive ability is likely to be extremely important to breeding programs. Finally, complex interactions are likely to be extremely important in terms of fitness and evolution. We are far from understanding plant competition, but we now have some sign posts that suggest how to get there.

\section{Literature Cited}

removing grasshoppers and other insects, the dominant herbivores, increased the importance of competition for some plants but decreased competition for others (Haag et al., 2004). Competition cannot be measured in isolation because it is completely contingent on the strength of other processes. These complex networks of interaction pose a 
Cahill, J.F. 2003. Lack of relationship between below-ground competition and allocation to roots in 10 grassland species. J. Ecol. 91:532540 .

Cahill, J.F., S.W. Kembel, and D.J. Gustafson. 2005. Differential genetic influences on competitive effect and response in Arabidopsis thaliana. J. Ecol. 93:958-967.

Campbell, B.D., J.P. Grime, and J.M.L. Mackey. 1991. A trade-off between scale and precision in resource foraging. Oecologia 87:532-538.

Darwin, C. 1859. The origin of species. John Murray, London.

Gaudet, C.L. and P.A. Keddy. 1988. A comparative approach to predicting competitive ability from plant traits. Nature 334:242-243.

Gaudet, C.L. and P.A. Keddy. 1995. Competitive performance and species distribution in shoreline plant communities: A comparative approach. Ecology 76:280-291.

Goldberg, D.E. 1990. Components of resource competition in plant communities, p. 27-49. In: Grace, J.B. and D. Tilman (eds.). Perspectives on plant competition. Academic Press, San Diego.
Grace, J.B. 2006. Structural equation modeling and natural systems. Cambridge University Press, UK.

Grime, J.P. 2001. Plant strategies, vegetation processes, and ecosystem properties. John Wiley and Sons, Chichester.

Haag, J.J., M.D. Coupe, and J.F. Cahill. 2004. Antagonistic interactions between competition and insect herbivory on plant growth. J. Ecol. 92:156-167.

Hodge, A. 2006. Plastic plants and patchy soils. J. Expt. Bot. 57:401-411.

Keddy, P.A. 2001. Competition. 2nd ed. Kluwer, Dordrecht.

Kembel, S.W. 2006. Leaf and root functional traits, phylogeny, and the structure of plant populations and communities. Department of Biology, University of Alberta, PhD Diss.

Kembel, S.W. and J.F. Cahill. 2005. Plant phenotypic plasticity belowground: A phylogenetic perspective on root foraging trade-offs. Amer. Nat. 166:216-230.

Lamb, E.G. and J.F. Cahill. 2006. Consequences of differing competitive abilities between juvenile and adult plants. Oikos 112:502-512.
Lamb, E.G., B.S. Shore, and J.F. Cahill. 2007. Water and nitrogen addition differentially impact plant competition in a native rough fescue grassland. Plant Ecology. (in press).

Robinson, D. 1994. The responses of plants to nonuniform supplies of nutrients. New Phytol. 127:635-674.

Rösch, H., M.W. VanRooyen, and G.K. Theron. 1997. Predicting competitive interactions between pioneer plant species by using plant traits. J. Veg. Sci. 8:489-494.

Shipley, B. 2000. Cause and correlation in biology. Cambridge University Press, UK.

Shipley, B. and D. Meziane. 2002. The balancedgrowth hypothesis and the allometry of leaf and root biomass allocation. Funct. Ecol. 16:326331.

Tilman, D. 1990. Constraints and tradeoffsToward a predictive theory of competition and succession. Oikos 58:3-15.

Williamson, G.B. 1990. Allelopathy, Koch's postulates, and the neck riddle, p. 143-162. In: Grace, J.B. and D. Tilman (eds.). Perspectives on plant competition. Academic Press, San Diego. 